\title{
Stabilization and Performance Improvement of Power-Hardware-in-the-Loop Test Using a Robust Data-Driven Method
}

\author{
Seyed Sohail Madani and Alireza Karimi
}

\begin{abstract}
Power-Hardware-in-the-Loop (PHIL) setups have gained high importance in validation of the performance of newly developed instruments and devices with low risk and implementation cost. However, the interconnection of a power hardware with a simulated model via a feedback loop using measured signals may make the closed-loop system unstable. In this paper, a systematic method is proposed to design a digital filter such that the closed-loop stability of the PHIL setup is guaranteed and its performance is optimized. The design of the filter is formulated as a specific controller synthesis problem that minimizes the deviation in measured feedback current and ensures a certain robustness margin. The design problem is written as a convex optimization and solved efficiently using available solvers. Due to the data-driven characteristic of the proposed method, there is no need for the model of the Hardware Under the Test (HUT) to guarantee stability and performance. Since the design is done in a systematic fashion using advanced control design techniques with a rigorous mathematical guarantee for stability, a high-performance filter can be designed with no need for laborious manual tuning that may lead to low-performance filters. This method is also extended to a multi-scenario case including different combinations of hardware and simulated systems. The PHIL experimental results validate the effectiveness of the proposed method while satisfying the required stability margin and improving the performance significantly compared to conventional filters.
\end{abstract}

Index Terms-Power-hardware-in-the-loop, data-driven control, robust control, stabilization, convex optimization.

\section{INTRODUCTION}

$\mathbf{H}$ ARDWARE-in-the-Loop (HIL) test is a flexible and efficient method to study the behavior of an instrument (i.e. HUT) and its interactions with the environment by measuring its states. HIL tests are usually conducted when a part of the system needs to be scaled-up [1] or when the objective is to study various normal and/or extreme and rare conditions [2]. The main objective of a HIL test is to be able to measure the states of the instrument with high fidelity. Thus, it is an important factor that the measurement data is not deviated. However, the most important factor is to make the

This project is carried out within the frame of the Swiss Centre for Competence in Energy Research on the Future Swiss Electrical Infrastructure (SCCER-FURIES) with the financial support of the Swiss Innovation Agency (Innosuisse - SCCER program). The work of S. S. Madani is supported by the Swiss National Science Foundation under Grant 200021_172828. (Corresponding author: Alireza Karimi.)

S. S. Madani (sohail.madani@epfl.ch) and A. Karimi (alireza.karimi@epfl.ch) are with the Laboratoire d' Automatique, École Polytechnique Fédérale de Lausanne, 1015 Lausanne, Switzerland.

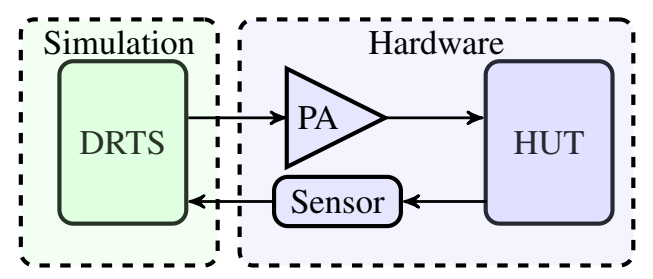

Fig. 1: PHIL general structure

system stable because in an unstable system no measurement is possible and the instruments may also be damaged. Since the measured signal is used in the feedback loop in the HIL setup, any filtering and process on this signal can directly affect the stability of the system. The main challenge is to condition, filter and process the measured signal such that the system remains stable while the quality of measurement data is not compromised.

A HIL setup can be seen as a complex measurement setup which is composed of different subsystems. When the environment is simulated by means of a Digital Real-Time Simulator (DRTS), different operating conditions can be tested with minimum cost and risk. If the HUT interacts with the DRTS via signals with no (or negligible) real power exchange and the controller is realized as the HUT, the HIL setup is called Control-Hardware-in-the-Loop (CHIL) [3].

In power systems, when a new component is added, there is a need for a series of tests to ensure the resiliency of the system. These tests should not impose high risk and cost. Using PHIL setups, different operation scenarios can be studied with reduced cost and time [4]. Moreover, in a tightly controlled laboratory environment, a PHIL setup can facilitate testing conditions which may be very extreme and/or dangerous in a power system [5]. In most applications, a PHIL setup is a HIL setup that includes DRTS and HUT which exchanges a considerable amount of power with the environment (e.g. when a real battery should be charged with a simulated synchronous generator) [6], [7]. In a PHIL setup, an interface between DRTS and HUT is required which basically includes a Power Amplifier (PA) and some sensors. PA can be realized either as a voltage amplifier or a current amplifier depending on the structure of the PHIL setup. However, in both cases, it is common to be denoted as PA in the PHIL literature. The general structure of a PHIL setup is shown in Fig. 1. In PHIL setups, DRTS should run with short and deterministic 
time steps which means that the time elapsed in the simulation is exactly equal to the time measured by a real-world clock [4]. In an ideal case, the PA and sensors should act as fixed gains in all frequencies with no delay and the simulation step time in DRTS should be zero, which are not realizable in practice [8], [9]. Moreover, depending on the combination of the system in simulation and HUT, the open-loop structure of the system may not necessarily be strictly proper which may result in high gain at high frequencies. The combination of the delay and the latter property may end up in PHIL instability that can put the HUT and PA under severe stress and cause damage [10]. As a remedy, the loop frequency response can be attenuated (more importantly at high frequencies) such that the closedloop system becomes stable. It can be realized by adding lowpass filters on measured signals, simulated impedances inside DRTS, physical impedances or a combination of those. However, these changes may result in the deviation of the measured value from what it should truly be. Different implementation methods and structures (a.k.a Interface Algorithms (IA)) are proposed to provide stability while trying to keep measurement signal deviation low. Some of the most well-known IAs are Ideal Transformer Model (ITM) [11], Time-variant First-order Approximation (TFA) [12], Transmission Line Model (TLM) [11], Partial Circuit Duplication (PCD) [11] and Damping Impedance Method (DIM) [10]. Among these methods, PCD, DIM and TLM are essentially based on the idea of using additional physical impedances which is not practically desirable because it is harder to implement, less flexible and has higher maintenance costs specifically in highpower applications [13]. Moreover, when the added hardware includes inductance, the performance in high frequencies is considerably affected [14]. TFA does not require an extra impedance, however, it has numerical stability problems and high sensitivity to noise [10]. The performance of some of the interface algorithms and their impact on the stability margin are compared in [15]. Among the mentioned methods, ITM is the best method in terms of implementation and is widely used in practice [9], [16]-[21]. However, stabilization is the main challenge using this method [10].

One of the main contributions of this paper is that the closed-loop stability of the PHIL setup is guaranteed without any knowledge about the parameters of the hardware. There are two important points, namely, stability guarantee and datadriven property.

Stabilization is the primary goal of almost all of the proposed methods in this area due to its safety hazards for the operators and the economic costs. Consequently, closedloop stability should be guaranteed in the design process. However, in contrast to most of the proposed methods, it is not enough to guarantee stability only for the nominal system. Uncertainty, as one of the main characteristics of PHIL systems, should also be considered in the proposed solution. The simulated system may not only have a single operating condition but instead a set of different possible conditions. For example, a power grid may have different typologies based on the status of the breakers or the HUT can have different possible modes which can be addressed by considering multimodel uncertainty. Moreover, the behaviour of the system may slightly and gradually change during the operation. For example, the resistive part may slightly change during a test which can be dealt with by a proper stability margin.

Using the measurement data directly for the design and bypassing the modeling step is another important characteristic of the proposed method. Conventionally, the data was used to construct a model (a.k.a system identification [22]) by an optimization and based on that model the controller was designed by another optimization. However, it should be assumed that the structure of the system is known (e.g. order) and a good low-order model can be obtained from the data which is not necessarily a valid assumption in practice. Specifically, in PHIL applications, it is not realistic to assume the availability of a reliable and accurate low-order model of the system because the unavailability of such a model is the major motivation behind conducting a PHIL experiment [13]. As an alternative, in direct-data-driven methods, the controller is designed directly from the data via only one optimization. In these methods, there is no need to put any assumption on the structure of the system which makes it suitable for PHIL applications. Basic direct-data-driven methods are reviewed in [23]. Recently, thanks to the advancements in solver technology and computation power, these methods have gained more attention [24]-[33]. The data used for synthesizing the controller may be in time-domain [24]-[26] or in frequency-domain [27]-[33]. One of the advantages of the latter is that the closed-loop sensitivity functions can be shaped directly in the frequency domain. However, in the application of controller design for PHIL setup, in addition to closed-loop functions, the controller itself should also be shaped which is not easily possible with the data-driven methods that are proposed in the literature.

Conventionally, in PHIL applications a parametric model (based on physical parameters of the system or identifying a low-order parametric model) of each element in the loop, such as PA, HUT, sensors, etc. are generated separately [34][36]. There are a limited number of researches addressing this problem by trying to use the data of PHIL systems [34], [37]-[41]. In [37]-[39], authors use the measured data and perform parametric identification of the input impedance of HUT. In [40], a similar method for impedance identification is used and a passivity criterion is considered for providing stability. However, for stabilization, the DIM method is used which has its own limitations as mentioned earlier. In [34], by considering the general structure of the system as a resistive voltage divider, it is assumed that the elements in the PHIL setup can be approximated by a first-order parametric model and the delay is approximated for a PHIL setup and then the stability is checked by the Routh criterion. Apart from the error introduced from the low order assumption and approximation of the delay, the studied structure is limited to a resistive voltage divider along with a very basic compensator that leads to low performance.

An efficient way to solve the design problem is to formulate it as a convex optimisation problem because there are very well-established numerical methods for convex programming which can handle a problem with a large number of variables and constraints [42]. Moreover, there are advanced solver technologies available to solve this type of problems (e.g. 
Simulation

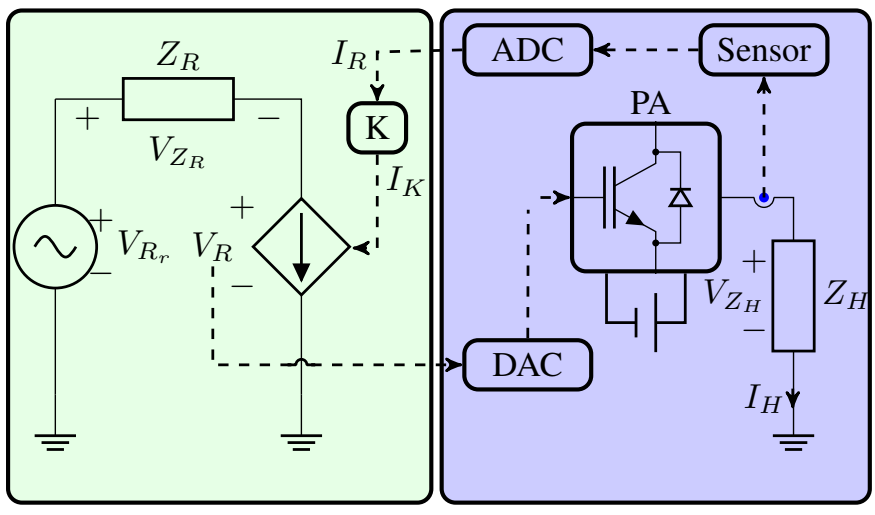

Fig. 2: PHIL circuit with ITM interface algorithm

[43]). Consequently, the main challenge is not to solve the convex programming problem, but instead, to formulate the practical problem at hand as a convex programming problem. To the best knowledge of the authors, there is no published literature that proposes a systematic, flexible and comprehensive controller design method for PHIL setups that guarantees closed-loop stability based on the data of the system without any assumption on the system structure (order, delay, etc.). In this paper, the problem of designing a high-performance filter that guarantees the closed-loop stability of a PHIL system is converted to a specific data-driven controller design method using convex optimization which can be solved fast and efficiently. It should be mentioned that the objective here is to put some constraints on the controller (filter) instead of the closed-loop transfer function which is usually done in the classical controller design methods. Only the frequency response of the loop is required to optimize the controller (filter) parameters under a predefined robust stability margin constraint. The proposed method is used to design a robust controller for the multi-scenario case as well.

The rest of the paper is organized as follows: In Section II, the structure of the PHIL setup is defined and in Section III, the controller design method using convex optimization is detailed. In Section IV, the process of data measurement and controller design for a case study is explained and finally in Section V, some concluding remarks are mentioned.

\section{PHIL SySTEM STRUCTURE}

ITM can be implemented using a current source or a voltage source on the PA side which are dual structures. In this paper, the voltage source ITM (simply denoted as ITM) is used and its general structure is depicted in Fig. 2, which is similar to the structure used in [4], [9], [10], [13], [21], [44], [45]. The results can be used straightforwardly for the current source ITM as well. The Thévenin theorem is applied in PHIL system analysis as the key modelling principle [46] and linear approximation of the system at different operating points enables the use of linear control system theory [4]. In fact, linear assumption and using linear techniques are common in the literature (e.g. [4]-[6], [13], [15], [15], [38], [44], [46][48]). However, multi-model structures or Linear Parameter Varying (LPV) methods [31], [49] can be used in some cases if this assumption is not valid. In the structure shown in Fig. 2, $V_{R_{r}}$ and $Z_{R}$ are equivalent voltage and impedance of the grid simulated in DRTS. This part is connected in series to a controlled current source which reflects the image of the current in the hardware side to the DRTS side. On the other hand, the voltage over the controlled current source (i.e. $V_{R}$ ) is amplified by PA to generate voltage $V_{Z_{H}}$, and, $Z_{H}$ represents the HUT.

Feedback current filtering is a well-known solution for stabilizing the closed-loop system [14], [45]. In this method, $K$ as an extra element is added to the loop in order to make it stable, while it should not deteriorate the signals and in an ideal case, it should act as a unity gain. In fact, the more $K$ is different from the unity the more deviation is imposed on the PHIL results. Besides, in the ideal case, PA should generate exactly what is asked for (i.e. $V_{Z_{H}}=V_{R}$ ). However, it does not happen due to the delay and non-ideal elements. The PHIL block diagram is shown in Fig. 3. In this figure, $G_{P}$ represents the transfer function of the PA while its input is the reference voltage and its output is $V_{Z_{H}}$ which includes the delay and usually has a low pass characteristic. $G_{H}$ represents the transfer function corresponding to $Z_{H}$ with its voltage and current as the input and output, respectively. $G_{S}$ denotes the transfer function of the current sensor. $K$ is the controller (filter) which acts on the feedback current in order to stabilize the closed-loop system. $G_{R}$ is the transfer function corresponding to $Z_{R}$ with the input $I_{K}$ and the output $V_{Z_{R}}$. DRTS is run at step $T_{R}$ which is limited by the DRTS technology and the complexity of the simulated system. Since the simulation in DRTS is in discrete-time and the hardware is in continuous-time, there is a need for ADC and DAC to connect these parts, which in the block diagram can be seen as a Zero-Order-Hold ( $\mathrm{ZOH})$ block and a sampler. One common method to assess the stability of the system is to model each part of the system separately and determine a simplified transfer function for the whole loop where some blocks may be ignored. For example, the dynamics of PA is ignored in [10] or modelled as a mixture of delay and a low-pass filter in [46]. For $G_{H}$, the first principles modeling (based on the assumption of known parameters and known physical equations governing the system) is common which is prone to uncertainty due to unknown parameters and physical interactions. Moreover, in many cases, the system is only analyzed in continuous-time ignoring the impact of the $\mathrm{ZOH}$ and the sampler. The mentioned issues may lead to an invalid understanding of the loop behavior which can end up in an incorrect stability analysis.

In this paper, a fully data-driven controller design method is proposed where the measured data is directly used for the design with no parametric identification step. To do so, the frequency response of the system from $V_{R}$ and to $I_{R}$ can be computed by applying a sine-sweep or a PseudoRandom Binary Sequence (PRBS) to $V_{R}$. This system is denoted as $G_{I H}$ which includes the $\mathrm{ZOH}, G_{P}, G_{H}, G_{S}$ and the sampler. Since $G_{R}$ is known from the simulation, the total 


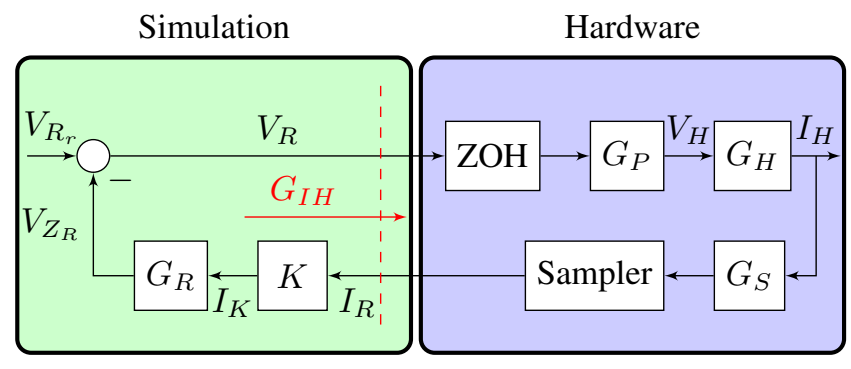

Fig. 3: PHIL setup block diagram

plant frequency response, $G$, can be considered as a series connection of $G_{I H}$ and $G_{R}$.

\section{Controller (Filter) Design}

In this section, a data-driven controller design method is introduced to design a low-pass filter for a PHIL system that guarantees the closed-loop stability and performance of the system in terms of accuracy and disturbance rejection. For the practical ease of implementation, the controller (filter) is defined in discrete-time, however, since the design method is in the frequency-domain, it can readily be rewritten for continuous-time controllers as well.

The system to be controlled is a Linear Time-Invariant (LTI) single-input single-output system. It is assumed that the frequency response of the system is computed based on a finite set of sampled measurement data and represented by a transfer function $G\left(e^{j \omega}\right) \in \mathbb{C}$ and is available for all $\omega \in \Omega=\left[-\omega_{n}, \omega_{n}\right]$, where $\omega_{n}=\pi / T_{s}$ is the Nyquist frequency and $T_{s}$ is the sampling period.

\section{A. Performance}

As mentioned previously, $K$ is an added element to the system to make the closed-loop system stable. However, it should not change the results of the experiment in an ideal case. Assume that the relative error between $I_{K}$ and $I_{R}$ is defined as follows:

$$
\frac{I_{K}-I_{R}}{I_{R}}=K-1
$$

which has a close connection to the ripple concept in filter design (see [50]). Consequently, the design problem can be defined as follows:

$$
\min _{K}\|K-1\|_{2}^{2}
$$

Note that, this type of objective is not common in the classical controller design methods, where usually a norm of a closedloop transfer function is minimized. Therefore, a standard $\mathcal{H}_{2}$ or $\mathcal{H}_{\infty}$ controller design method cannot be used here. Using the definition of the two-norm of a transfer function, it can be shown that the problem (2) is equivalent to

$$
\min _{K} \int_{-\omega_{n}}^{\omega_{n}}\left|K\left(e^{j \omega}\right)-1\right|^{2} d \omega
$$

In order to have an upper bound on the gain of the filter, the following constraint can be used:

$$
\left|K\left(e^{j \omega}\right)\right|<W_{u}(\omega) \quad \forall \omega \in \Omega
$$

Similarly, a lower bound is required to guarantee the signal passes up to a predefined value which can be written as:

$$
\left|K\left(e^{j \omega}\right)\right|>W_{l}(\omega) \quad \forall \omega \in \Omega
$$

In addition, it is clear that the result of this optimization does not necessarily stabilize the closed-loop system. Particularly, since $G$ is not necessarily strictly proper and may have a very large gain at high frequencies, for closed-loop stability the controller (filter) $K$ should have frequency roll-off at high frequencies. In the following part, a constraint for stability margin will be discussed.

\section{B. Robustness}

In order to make the system robustly stable, a guaranteed stability margin is crucial. In this paper, the modulus margin $(M)$ has been used as the stability margin which guarantees the well-known gain margin and phase margin simultaneously. Modulus margin is the minimum distance of the open-loop transfer function from the critical point (i.e. $-1+0 j$ ) in the Nyquist diagram, which can be defined as:

$$
M=\left(\sup _{\omega}\left|\mathcal{S}\left(e^{j \omega}\right)\right|\right)^{-1}
$$

where $\mathcal{S}=(1+L)^{-1}$ is the output sensitivity function and $L=G K$ is the open-loop transfer function. For a closed-loop stable system, the modulus margin can be guaranteed by:

$$
\left|\mathcal{S}\left(e^{j \omega}\right)\right|<M_{l}^{-1} \quad \forall \omega \in \Omega
$$

where $M_{l}$ is the desired lower-bound for the modulus margin $M$. The above constraint guarantees a minimum distance from the critical point in the Nyquist diagram but it does not guarantee that the Nyquist diagram will not turn around the critical point. This problem is studied at the end of this section.

\section{Disturbance Rejection}

In addition to the previous constraints, the output disturbance rejection can also be considered as a performance in the controller design. The transfer function from the output disturbance to $I_{K}$ is $\mathcal{U}=K(1+L)^{-1}$, where $\mathcal{U}$ is the input sensitivity function and its magnitude can be limited using the following constraint:

$$
\left|\mathcal{U}\left(e^{j \omega}\right)\right|<U_{u} \quad \forall \omega \in \Omega
$$

where $U_{u}$ is the desired upper bound for the input sensitivity function.

\section{Controller Design Problem}

The controller design problem can be cast as the following nonlinear constrained optimization problem:

$$
\begin{array}{cl}
\min _{K} & \int_{-\omega_{n}}^{\omega_{n}}(K-1)(K-1)^{*} d \omega \\
\text { s.t. } & K K^{*}<W_{u} W_{u}^{*} \quad \forall \omega \in \Omega \\
& K K^{*}>W_{l} W_{l}^{*} \quad \forall \omega \in \Omega \\
& \mathcal{S S}^{*}<M_{l}^{-2} \quad \forall \omega \in \Omega \\
& \mathcal{H U}^{*}<U_{u}^{2} \quad \forall \omega \in \Omega .
\end{array}
$$


In this optimization problem, the objective (i.e. (9a)) is equivalent to (3). The first and the second constraint (i.e. (9b) and (9b)) are equivalent to (4) and (5), respectively. The third constraint (i.e. (9d)) is equivalent to (7) and provides a predefined stability margin. And finally, the fourth constraint (i.e. (9e)) is equivalent to (8) and is added to limit the magnitude of the transfer function from the disturbance to the $I_{K}$ in order not to let the measurement noise and disturbance be amplified in the simulation. Basically, from the control point of view, the input and output closed-loop sensitivity functions (i.e. $\mathcal{S}$ and $\mathcal{U}$ ) act toward disturbance rejection and help the quality of the signals not to be affected by disturbance and noise. For the sake of simplicity in notation, the functions' arguments are omitted in the above problem and in the rest of the paper and are only reiterated when deemed necessary. This problem is a non-standard control problem, non-convex and with an infinite number of constraints. In the sequel, the steps required to change this problem into a convex optimization are explained.

1) Controller Structure and problem formulation: The controller is defined as:

$$
K(z)=\frac{X(z)}{Y(z)}
$$

where both $X$ and $Y$ are polynomials in $z$ as follows:

$$
\begin{aligned}
& X(z)=\left(x_{0}+x_{1} z+\ldots+x_{n_{K}} z^{n_{K}}\right) \\
& Y(z)=\left(y_{0}+y_{1} z+\ldots+y_{n_{K}-1} z^{n_{K}-1}+z^{n_{K}}\right)
\end{aligned}
$$

where $x_{i}$ for $i=0,1, \ldots, n_{K}$ and $y_{i}$ for $i=0,1, \ldots, n_{K}-1$ are the controller parameters. Let $Q=X-Y$ and $P=Y+$ $G X$, then obviously

$K-1=\frac{Q}{Y}, \mathcal{S}=\frac{1}{1+\frac{G X}{Y}}=\frac{Y}{P}$, and, $\mathcal{U}=\frac{\frac{X}{Y}}{1+\frac{G X}{Y}}=\frac{X}{P}$.

Consequently, problem (9) can be written as:

$$
\begin{aligned}
\min _{X, Y} & \int_{-\omega_{n}}^{\omega_{n}}\left(\frac{Q}{Y}\right)\left(\frac{Q}{Y}\right)^{*} d \omega \\
\text { s.t. } & \left(\frac{X}{Y}\right)\left(\frac{X}{Y}\right)^{*}<W_{u} W_{u}^{*} \quad \forall \omega \in \Omega \\
& \left(\frac{X}{Y}\right)\left(\frac{X}{Y}\right)^{*}>W_{l} W_{l}^{*} \quad \forall \omega \in \Omega \\
& \left(\frac{Y}{P}\right)\left(\frac{Y}{P}\right)^{*}<M_{l}^{-2} \quad \forall \omega \in \Omega \\
& \left(\frac{X}{P}\right)\left(\frac{X}{P}\right)^{*}<U_{u}^{2} \quad \forall \omega \in \Omega
\end{aligned}
$$

This problem is still non-convex and its convexification is explained in the next part.

2) Convex Controller Design Problem: It can be shown that (12a) is equivalent to:

$$
\begin{aligned}
& \min _{X, Y} \int_{-\omega_{n}}^{\omega_{n}} \gamma(\omega) \\
& \text { s.t. } \quad\left(\frac{Q}{Y}\right)\left(\frac{Q}{Y}\right)^{*}<\gamma(\omega) \quad \forall \omega \in \Omega
\end{aligned}
$$

where $\gamma(\omega)>0$ for all $\omega \in \Omega$ is an unknown function. Moreover, as a general procedure, constraints of the problem
(12) are written in the convex-concave form and then, the concave part is linearized around an initial stabilizing controller $K_{c}(z)=X_{c}(z) Y_{c}^{-1}(z)$ to obtain a convex constraint. To start with (12b), it can be written as:

$$
X X^{*}-W_{u} Y Y^{*} W_{u}^{*}<0 \quad \forall \omega \in \Omega .
$$

It should be noted that left hand side of (14) has quadratic from in $X$ and $Y$ and according to (11), $X$ and $Y$ are linear in controller parameters (i.e. $x_{i}$ for $i=0, \ldots, n_{K}$ and $y_{i}$ for $\left.i=0, \ldots, n_{K}-1\right)$.

Let $F:=W_{u} Y$ and $F_{c}:=W_{u} Y_{c}$. By developing $(F-$ $\left.F_{c}\right)\left(F-F_{c}\right)^{*} \geq 0$, it can be shown that:

$$
X X^{*}-F_{c} F^{*}-F F_{c}^{*}+F_{c} F_{c}^{*} \leq 0 \quad \forall \omega \in \Omega
$$

is a sufficient convex constraint for (14). Similarly, other constraints in the problem can be convexified.

3) Semi-definite Controller Design Problem: In general, the frequency response that is obtained from the sampled data can be calculated as [51]:

$$
G\left(e^{j \omega}\right)=\frac{\sum_{k=0}^{N-1} y(k) e^{-j \omega T_{s} k}}{\sum_{k=0}^{N-1} u(k) e^{-j \omega T_{s} k}}
$$

where $u(k)$ is the input and $y(k)$ is the output which are assumed equal to zero for $k \geq N$ and $k<0$. As it can be seen, this function is continuous in frequency and can be calculated at any arbitrary frequency. If the plant obtained in (16) is used to construct the problem in (12), it will be a semi-infinite programming (i.e. with an infinite number of constraints). In order to make the problem tractable, the frequency response can be sampled at a finite set of frequencies, $\Omega_{N}=\left\{\omega_{1}, \ldots, \omega_{N}\right\}$ (since the constraints are symmetric, non-negative frequencies are considered). As a result, the semi-infinite problem would be changed to a tractable semi-definite programming. Following the same convexification procedure for all of the constraints, the controller design problem can be written as a semi-definite programming as follows:

$$
\begin{array}{cl} 
& \min _{X, Y} \sum_{\Omega_{N}} \gamma(\omega) \\
\text { s.t. } & Q \gamma^{-1}(\omega) Q^{*}-Y_{c} Y^{*}-Y Y_{c}^{*}+Y_{c} Y_{c}^{*}<0 \\
& X X^{*}-F_{c} F^{*}-F F_{c}^{*}+F_{c} F_{c}^{*}<0 \\
& W_{l} Y Y^{*} W_{l}^{*}-X_{c} X^{*}-X X_{c}^{*}+X_{c} X_{c}^{*}<0 \\
& Y Y^{*} M_{l}^{2}-P_{c} P^{*}-P P_{c}^{*}+P_{c} P_{c}^{*}<0 \\
& X X^{*} U_{u}^{-2}-P_{c} P^{*}-P P_{c}^{*}+P_{c} P_{c}^{*}<0
\end{array}
$$

for all $\omega$ in $\Omega_{N}$ where $P_{c}=Y_{c}+G X_{c}$. In this algorithm, $\gamma\left(\omega_{1}\right)$ to $\gamma\left(\omega_{N}\right)$ are $N$ positive dummy variables that make the objective function linear. Constraint set (17b) using Schur complement lemma has convex form and constraint sets (17c)(17f) have quadratic-convex from. Consequently, this problem has a linear objective function with convex constraints which is a convex programming and can be solved efficiently and fast by available convex-optimization solvers. Problem (17) is obtained based on the convexification of the original problem around the initial controller. Consequently, even though the stability will be kept anyway, the performance will highly depend on the choice of the initial controller. To improve the 
performance an iterative approach can be used that considers the optimal controller from the previous iteration as the initial controller for the next iteration. This way, the stability property will be preserved while the performance will converge to that of a local minimum or a saddle point of the original nonconvex problem [30].

4) Closed-loop stability: If $P_{c} P^{*}+P P_{c}^{*}>0, Y \neq 0$ and $Y_{c} \neq 0$ for all $\omega \in \Omega$ and the order of $Y$ equals the order of $Y_{c}$, then the number of encirclements of $G K$ around the critical point will be equal to that of $G K_{c}$ (see Theorem 2 in [30] for multivariable systems). It can be shown that (17e) or (17f) ensures that $P_{c} P^{*}+P P_{c}^{*}>0$ and (17b) is sufficient for $Y \neq 0$ and $Y_{c} \neq 0$. Consequently, the closed-loop system will be stable if the initial controller $K_{c}$ is stabilizing and the order of $Y$ equals the order of $Y_{c}$. It should be noted that assuming the availability of an initial stabilizing controller is not a strong assumption for stable plants since a sufficiently small gain always stabilizes the output-feedback closed-loop system.

\section{E. Controller Design for Multi-Scenario case}

In this section, the problem of encountering different scenarios in the PHIL setup is addressed. For example, when different topologies of a grid or testing more than one type of HUT are considered. All these scenarios may amount to different $Z_{R}$ and $Z_{H}$ which eliminates all the stability and performance guarantees. As the number of scenarios increases, designing without a systematic method becomes more time consuming and may lead to an unnecessary conservative result. Assume there are $n_{G}$ scenarios each of which corresponds to one PHIL setup configuration (i.e. one $\left(Z_{R}, Z_{H}\right)$ ). The set of all models is defined as $\mathcal{G}=\left\{G_{1}, G_{2} \ldots, G_{n_{G}}\right\}$ where $G_{i}$ denotes frequency response corresponding to $i$-th scenario. Consequently, (17) can be extended to:

$$
\begin{array}{ll} 
& \min _{X, Y} \sum_{\Omega_{N}} \gamma(\omega) \\
\text { s.t. } & Q_{i} \gamma^{-1}(\omega) Q_{i}^{*}-Y_{c} Y^{*}-Y Y_{c}^{*}+Y_{c} Y_{c}^{*}<0 \\
& X X^{*}-F_{c} F^{*}-F F_{c}^{*}+F_{c} F_{c}^{*}<0 \\
& W_{l} Y Y^{*} W_{l}^{*}-X_{c} X^{*}-X X_{c}^{*}+X_{c} X_{c}^{*}<0 \\
& Y Y^{*} M_{l}^{2}-P_{c i} P_{i}^{*}-P_{i} P_{c i}^{*}+P_{c i} P_{c i}^{*}<0 \\
& X X^{*} U_{u}^{-2}-P_{c i} P_{i}^{*}-P_{i} P_{c i}^{*}+P_{c i} P_{c i}^{*}<0
\end{array}
$$

for all $\omega$ in $\Omega_{N}$ and for all $G_{i}$ in $\mathcal{G}$.

\section{CAse Study}

The controller design problem explained in Section III is applied on a PHIL setup. In this case study, Opal-RT OP5600 and Puissance plus AC amplifier PA-3x1000-AB/260V-7.7A are used as DRTS and PA, respectively. The PHIL test setup is shown in Fig. 4. It should be noted that the results presented in this section do not aim to reflect the characteristics of any specific system or device. In this case study, resistiveinductive impedances for both $Z_{R}$ and $Z_{H}$ are selected which is common in PHIL system studies (e.g. [6], [21], [44], [52]). For the base-case scenario, $L_{R}=9 \mathrm{mH}, L_{H}=6 \mathrm{mH}$, and $R_{R}=R_{H}=11 \Omega$. It should be emphasized again that these

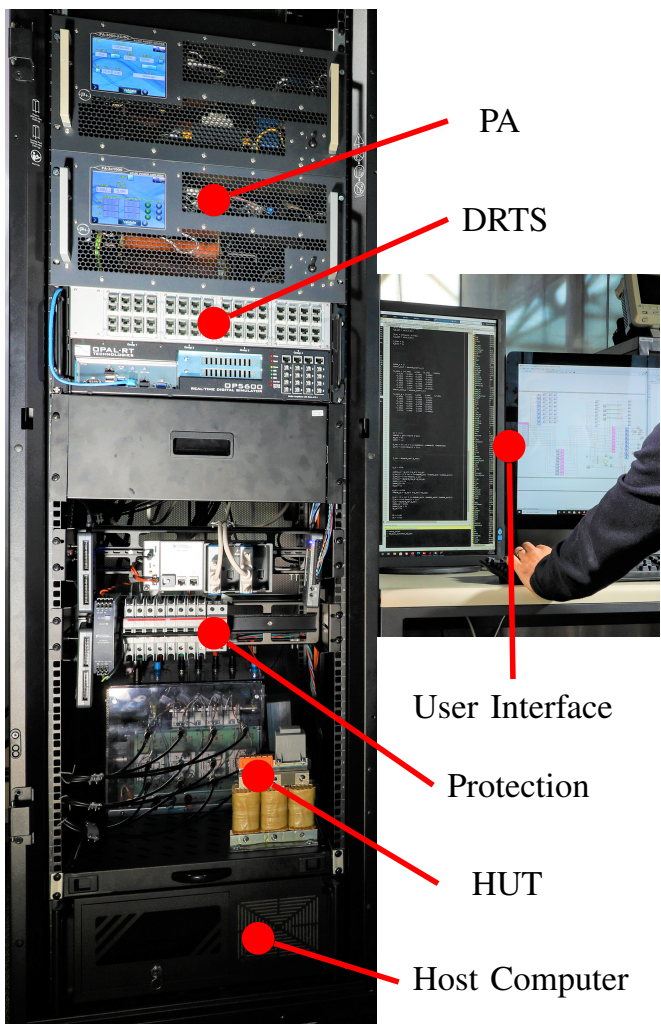

Fig. 4: PHIL setup

parameters are used neither for the stability analysis nor for the design and are just mentioned for the completeness of the representation.

\section{A. Data Measurement}

A PRBS signal with order 10 and magnitude of $10 \mathrm{~V}$, which is generated in DRTS is used to excite the system and the response of the system is logged. In practice, the measurement data is contaminated with noise whose impact on the frequency response can be considered with an additive uncertainty as $\tilde{G}\left(e^{j \omega}\right)=G\left(e^{j \omega}\right)+W_{u}\left(e^{j \omega}\right) \Delta$ where $W_{u}\left(e^{j \omega}\right)$ is the uncertainty filter and $\Delta$ is the uncertainty disk [53]. $W_{u}$ can be calculated using an estimate of the noise spectrum from the input-output data [22]. A very practical method to reduce the impact of the noise is to use periodic signals [51]. In this case study, 4 periods of measured data are used to calculate the frequency responses. In Fig. 5, one period of the excitation signal and the measured $I_{R}$ are shown. It can be seen that based on the order of the PRBS signal and the sampling time of DRTS, the whole data measurement process takes less the one second. The Fourier transform of input and output data is calculated and $G_{I H}$ is obtained. Since $Z_{R}$ is known, its frequency response and consequently the frequency response of the plant $G$ can be obtained as shown in Fig. 6. In Fig. 7, the Nyquist diagram shows one encirclement of the critical point, meaning that without a controller (filter) the PHIL becomes unstable. 


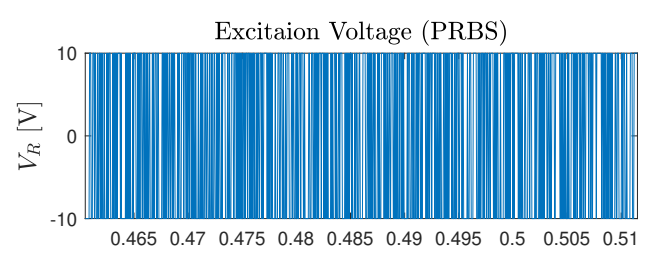

Measured Current

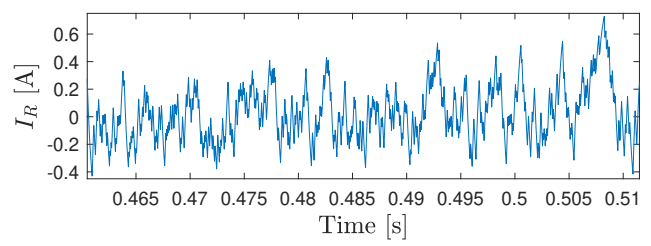

Fig. 5: Excitation at $V_{R}$ and measured $I_{R}$

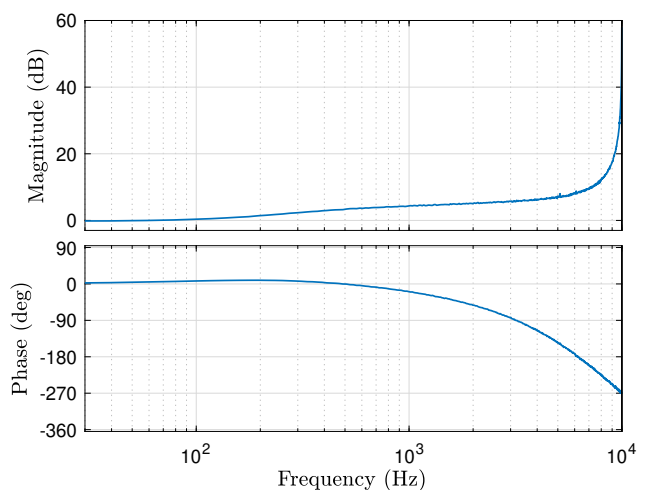

Fig. 6: Bode plot of $G$

\section{B. Controller (filter) Design}

Finding a stabilizing controller for this system is very easy since even a small gain provides closed-loop stability. However, this is not practical since for $K \ll 1$, the current $I_{K} \ll I_{R}$ and the precision would be very low. In the literature, a common practice is to use a simple first-order ( [9], [13], [19], [20], [44]-[47]) or a second-order Butterworth ( [5], [48]) low-pass filter for PHIL stabilization.

As the first step, the limitations of the conventional lowpass filtering method is studied to see what can be achieved using the simple filters proposed in the literature. Assume that a set of first and second-order low-pass Butterworth filters

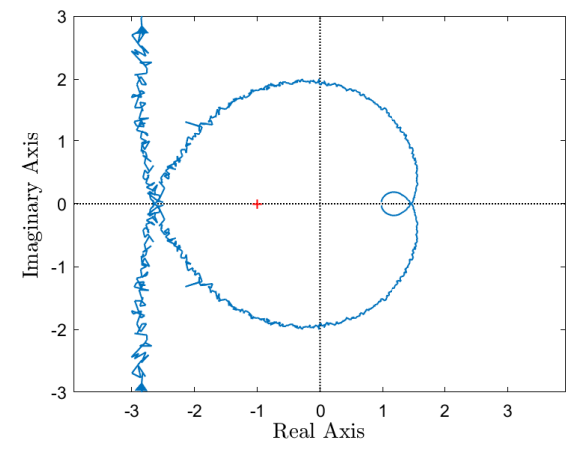

Fig. 7: Nyquist plot of $G$
TABLE I: Controller Design parameters

\begin{tabular}{cc|cc}
\hline \hline Param. & Value & Freq. [Hz] & $W_{l}$ \\
\hline$M_{l}$ & 0.5 & {$[0,50]$} & 0.9999 \\
$N$ & 511 & $(50,300]$ & 0.999 \\
$U_{u}$ & $-3 \mathrm{~dB}$ & $(300,900]$ & 0.99 \\
$n_{K}$ & 17 & $(900,1800]$ & 0.9 \\
$W_{u}$ & $2-W_{l}$ & $\left(1800,1 / 2 T_{s}\right]$ & 0 \\
\hline \hline
\end{tabular}

are available with bandwidth varying from $0.5 \mathrm{kHz}$ to $2 \mathrm{kHz}$ with steps of $0.5 \mathrm{kHz}$. The Nyquist plots of the open-loop transfer functions using these filters are shown in Fig. 8a. It can be seen that for both first-order and second-order filters the Nyquist diagram approaches the critical point as the bandwidth of the filter increases. In order to assess the stability margin and output disturbance rejection in the control signal, the bode diagrams of the output and input sensitivity functions are depicted in Fig. 8b and Fig. 8c, respectively. As shown in Fig. 8, there is a trade-off between the bandwidth of the controller and the stability margin. In addition, it is very important to note that simply increasing the order of the filter affects the stability margin adversely. Consequently, the stability issue is even more critical when the order is increased (e.g. see Fig. 8a and Fig. 8b). In other words, when there is no systematic method to take advantage of the added capability and when the added complexity is not dealt with correctly, it may end up in the system's instability. The lack of a systematic, efficient, and, advanced design method may be the main reason why in the current literature the order is kept low. Considering the mentioned items, a first-order filter (which was used also in [9], [13], [19], [20], [44]-[47]) with a bandwidth of $1250 \mathrm{~Hz}$, denoted by $K_{0}$, is selected and compared with our controller in the sequel.

For designing the controller, the problem (17) is solved using MOSEK [43] in less than 20 seconds on a conventional desktop computer. A fast systematic design method is very valuable, compared with the manual tuning when a large number of tests should be carried out in a short time. The design parameters are listed in Table I and the designed controller is denoted as $K_{D}$. The Nyquist diagram of systems using $K_{0}$ and $K_{D}$ are compared in Fig. 9. It can be seen that both controllers have made the closed-loop system stable. However, $K_{D}$ has provided a farther distance from the critical point, which results in higher robustness ( $M=0.5$ for $K_{D}$ vs. $M=0.39$ for $K_{0}$ ). The disturbance rejection performance of the closed-loop systems are compared in Fig. 10. As shown in the figure, the input sensitivity function has not violated the required limit and provides better disturbance attenuation compared to $K_{0}$. Finally, the frequency response of $K_{D}$ is compared with that of $K_{0}$ in Fig. 11, which shows considerable improvement. Fig. 12 clearly shows the impact of the constraints on the controller. In order to have a better overview, the regions satisfying different accuracies are listed in Table II. It can be seen that requirement set by the constraints are met and the performance compared to the conventional filter are improved significantly. To validate the performance of the proposed method, the designed controller is implemented on the PHIL setup and a sinusoidal sweep signal is applied as 


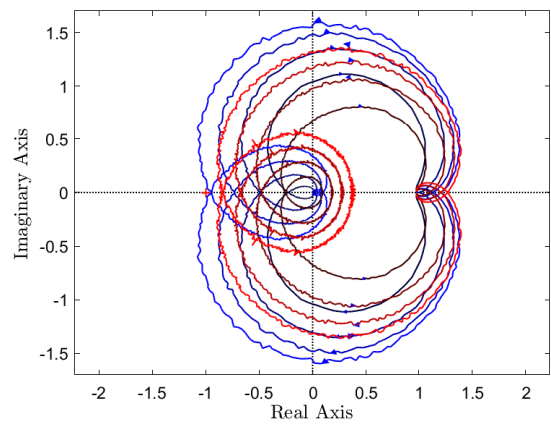

(a) Nyquist plot of the open-loop systems

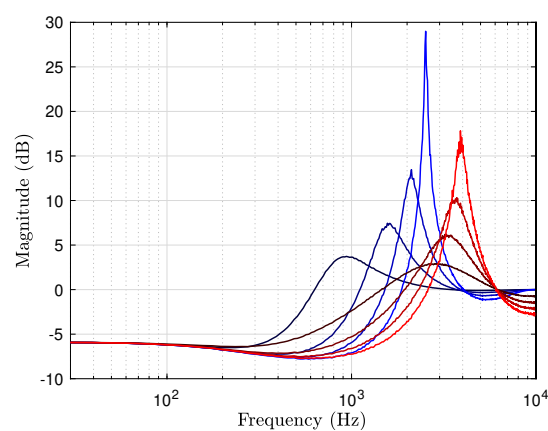

(b) Bode magnitude plot of the output sensitivity

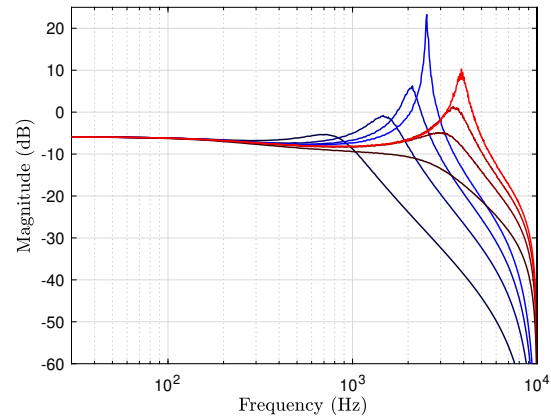

(c) Bode magnitude plot of the input sensitivity

Fig. 8: Systems using second-order (blue) and first-order (red) filters (dark to light represents $0.5 \mathrm{KHz}$ to $2 \mathrm{kHz}$ bandwidth)

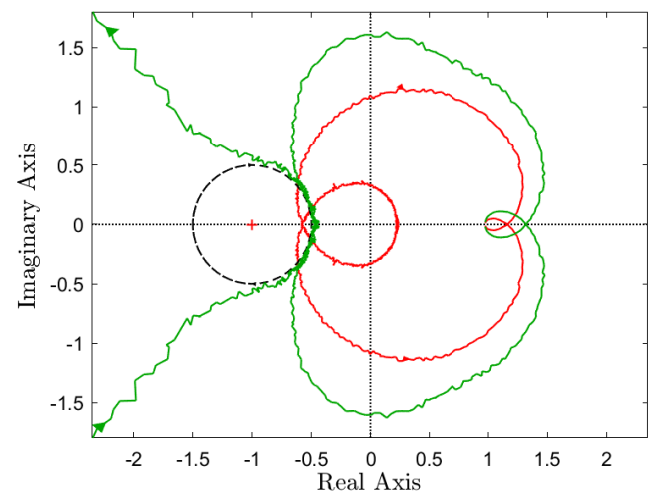

Fig. 9: Nyquist plot of the open-loop systems using $K_{0}$ (red) and $K_{D}$ (green), (corresponding constraint (dashed-black))

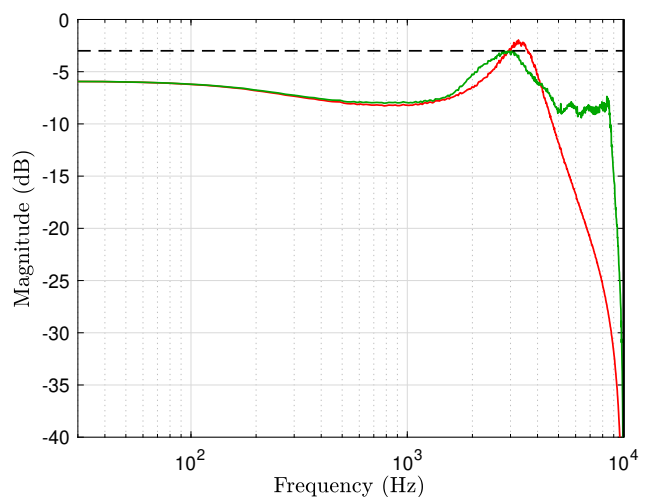

Fig. 10: Bode magnitude plot of the input sensitivity for systems using $K_{0}$ (red) and $K_{D}$ (green), (corresponding constraint (dashed-black))

$V_{R_{r}}$. Fig. 13 compares $I_{R}$ and $I_{K}$ at different frequencies. In the fundamental frequency (i.e. $50 \mathrm{~Hz}$ ), $K_{D}$ almost perfectly tracks while $K_{0}$ has a slight deviation. In $300 \mathrm{~Hz}$, the deviation using $K_{0}$ is visible. The difference at $900 \mathrm{~Hz}$ is considerable while at $1800 \mathrm{~Hz}$, the response of $K_{0}$ is significantly deviated.

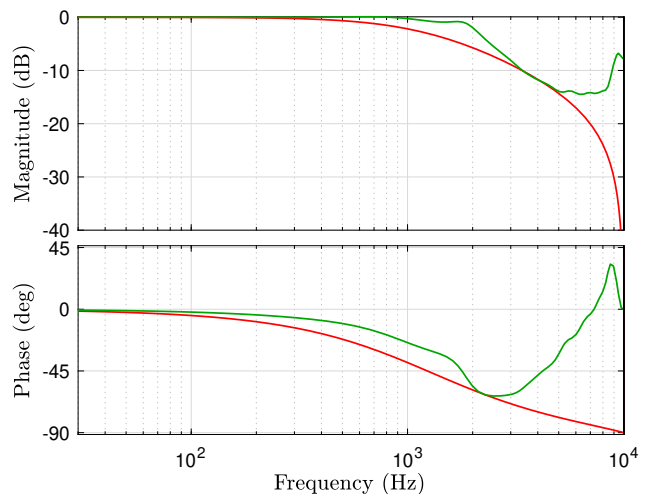

Fig. 11: Bode plot of the controllers $K_{0}$ (red) and $K_{D}$ (green)

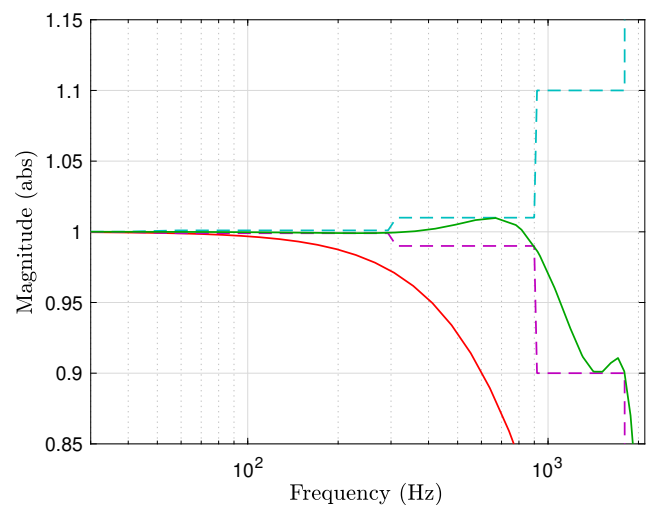

Fig. 12: Bode magnitude plot of controllers $\left(K_{D}\right.$ (green), $K_{0}$ (red), $W_{l}$ (dashed magenta), $W_{u}$ (dashed cyan))

TABLE II: Frequency regions for different error bounds

\begin{tabular}{cccc}
\hline \hline $\max |K-1|$ & $\begin{array}{c}\text { Up to Freq. } \\
\left(K_{0}\right)\end{array}$ & $\begin{array}{c}\text { Up to Freq. } \\
(\text { Expected) }\end{array}$ & $\begin{array}{c}\text { Up to Freq. } \\
\left.\text { (realized by } K_{D}\right)\end{array}$ \\
\hline & {$[\mathrm{Hz}]$} & {$[\mathrm{Hz}]$} & {$[\mathrm{Hz}]$} \\
\hline 0.0001 & 8 & 50 & 67 \\
0.001 & 55 & 300 & 380 \\
0.01 & 177 & 900 & 900 \\
0.1 & 601 & 1800 & 1800 \\
\hline \hline
\end{tabular}


TABLE III: Multi scenario system parameters $\left(L_{R}[\mathrm{mH}]\right.$, $\left.L_{H}[\mathrm{mH}], R_{R}[\Omega], R_{H}[\Omega]\right)$

\begin{tabular}{c|c||c|c||c|c||c|c}
\hline \hline Sc. & Param. & Sc. & Param. & Sc. & Param. & Sc. & Param. \\
\hline 1 & $(3,3,11,22)$ & 5 & $(3,3,11,11)$ & 9 & $(3,6,11,22)$ & 13 & $(3,6,11,11)$ \\
\hline 2 & $(9,3,11,22)$ & 6 & $(9,3,11,11)$ & 10 & $(9,6,11,22)$ & 14 & $(9,6,11,11)$ \\
\hline 3 & $(3,3,22,22)$ & 7 & $(3,3,22,11)$ & 11 & $(3,6,22,22)$ & 15 & $(3,6,22,11)$ \\
\hline 4 & $(9,3,22,22)$ & 8 & $(9,3,22,11)$ & 12 & $(9,6,22,22)$ & 16 & $(9,6,22,11)$ \\
\hline \hline
\end{tabular}

\section{Discussion on design parameters and problem constants}

Because the proposed method is systematic and data-driven, unlike most methods in the literature, it is independent of the parameters of the problem. These values are selected as typical values just to provide a showcase. In this paper, two distinct sets of fixed values can be seen.

1) System constants: These constants stem from the PHIL equipment and are not selected by the controller designer. For example, $T_{s}$ depends on the computation power of the DRTS and is selected to be $50 \mu \mathrm{s}$ which is a typical value [54]. As another example, $L_{R}$ and $R_{R}$ depend on the simulated grid and $L_{H}$ and $R_{H}$ are based on the HUT. In this paper, the grid is assumed to be a distribution system in which the $X / R$ is relatively small. As the base case, the $X / R-$ ratio of the impedances in HUT and DRTS belong to the interval $[0.15,0.26]$ so do the lines $4 c-70$ and $4 c-95-S A C-A C$ of the IEEE of European low voltage test feeder [55].

2) Design parameters: These parameters are selected by the controller designer based on the expected performance. For example, the modulus margin is selected based on the required robustness and is selected to be $-6 d B(\approx 0.5)$ which is a typical value in the controller design. $N$ is the number of sampled points in the frequency-domain which can be chosen relatively large without severe impact on the solver time [30]. $U_{u}$ can be selected based on the measurement noise level and is simply chosen to be $-3 d B(\approx 0.7)$. If the measurement is highly noisy, this value can be set lower. $n_{K}$ determines the flexibility of the controller, and in this problem, with $n_{K}=17$ a good performance has been achieved. $W_{l}$ and $W_{u}$ determine maximum deviation in the measured current and for the sake of simplicity, are set to be symmetric. In this paper, it has been required that $|K-1|$ must be less than 0.0001 up to the first harmonic, less than 0.001 up to the 6th harmonic, less than 0.01 up to the 18 th harmonic, and, less than 0.1 up to the 36 th harmonic. These values are selected as an example showing how the design requirements can be met by this method. In practice, these values should be selected based on the desired performance of the problem.

\section{Multi-Scenario Case}

In this part, 16 different scenarios are considered whose corresponding parameters are listed in Table. III and frequency responses are shown in Fig. 14. The problem (18) is solved considering all scenarios as one comprehensive programming problem and the designed controller is denoted as $K_{D M}$. For this design, a guarantee for $|K-1|$ to be less than 0.001 up to the first harmonic, less than 0.01 up to the 6th harmonic, and, less than 0.1 up to the 12th harmonic is considered. The Nyquist diagram of systems using the $K_{0}$ and $K_{D M}$ are
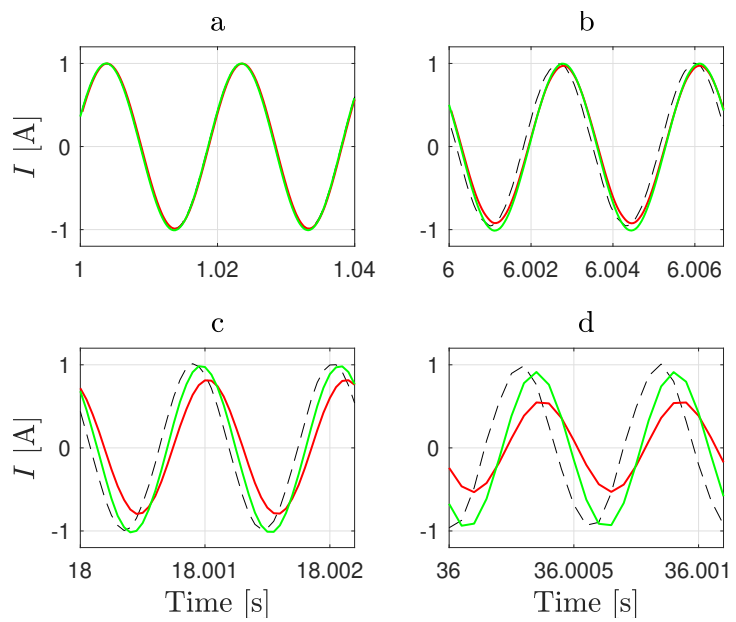

Fig. 13: Current measured from PHIL setup ( $I_{R}$ (black dashed), $I_{K}$ with $K_{D}$ (solid green), $I_{K}$ with $K_{0}$ (solid red)) a, b, c, and d, correspond to $50 \mathrm{~Hz}, 300 \mathrm{~Hz}, 900 \mathrm{~Hz}$, and $1800 \mathrm{~Hz}$, respectively.

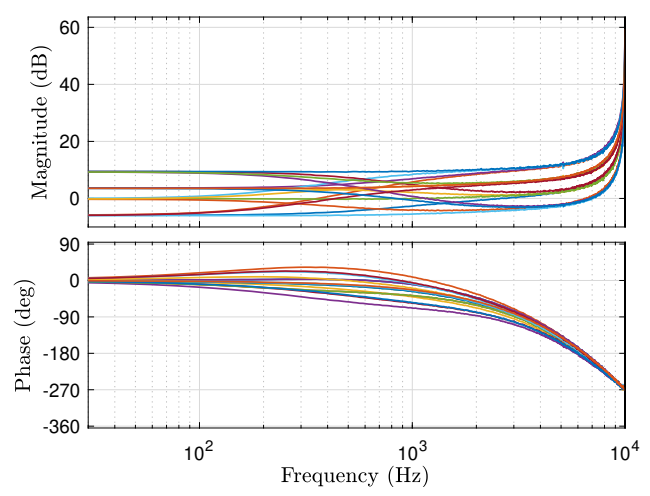

Fig. 14: Bode plot of $G_{i}$ in different scenarios

compared in Fig. 15 which shows that $K_{0}$ cannot stabilize all scenarios, while $K_{D M}$ stabilizes all scenarios and provides $M=0.5$ for all of them. Fig. 16, shows that $K_{D M}$ has satisfied the required attenuation for all scenarios. If one wants to use the conventional method to design a controller which stabilizes all models with comparable robustness and disturbance rejection (named as $K_{0 M}$ ), the bandwidth should be decreased to $400 \mathrm{~Hz}$, which results in losing performance. In Fig. 17, the bode diagram of $K_{0 M}$ is compared with $K_{D M}, K_{D}$ and $K_{0}$. As expected, the performance of $K_{D M}$ is limited compared to $K_{D}$ since challenging scenarios are included (e.g. scenario No. 8 and scenario No. 6). However, compared to the $K_{0 M}$, it shows considerable improvement. As an example, scenario No. 8 is tested in PHIL setup using $K_{D}$ and $K_{D M}$ and the results are shown in Fig. 18. As expected, $K_{0}$ destabilizes the system while $K_{D M}$ stabilizes and shows good performance.

\section{CONCLUSION}

In this paper, the problem of PHIL setup stabilization and performance improvement has been addressed and a datadriven controller design method has been proposed. In this method, in order to reduce the impact of the added controller 


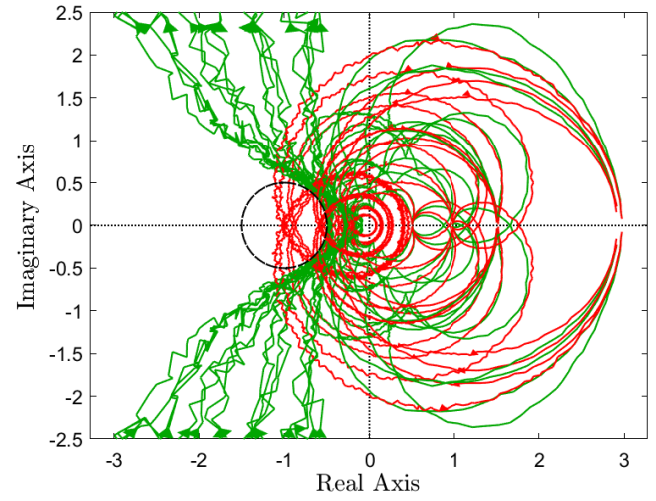

Fig. 15: Nyquist plot of open-loop systems corresponding to different scenarios by using $K_{0}$ (red) and $K_{D M}$ (green)

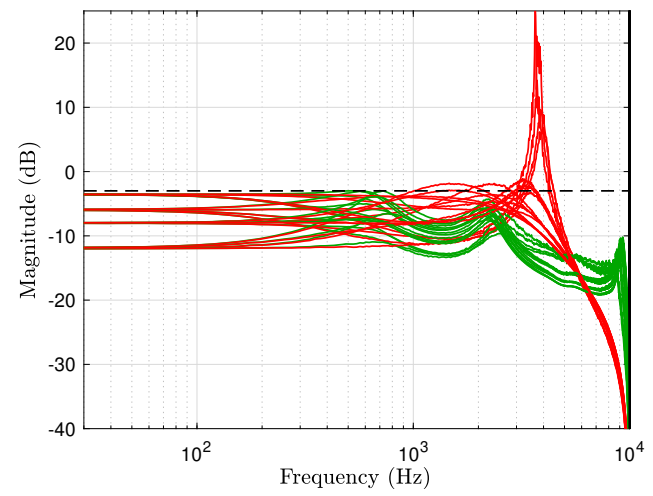

Fig. 16: Bode magnitude plot of input sensitivity of systems corresponding to different scenarios by using $K_{0}$ (red) and $K_{D M}$ (green)

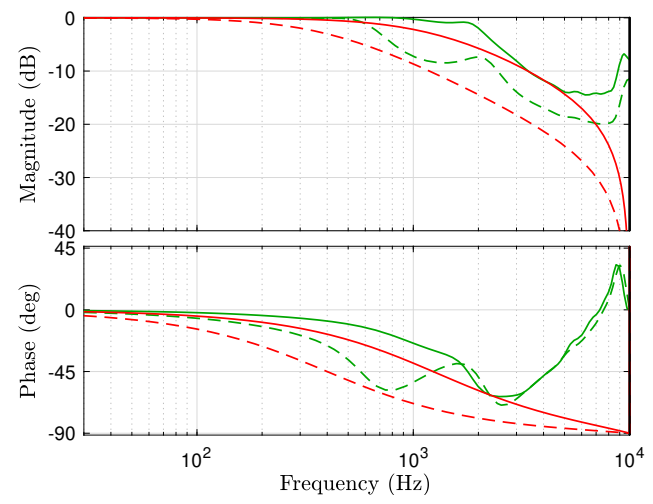

Fig. 17: Bode of the controllers, $K_{0 M}$ (dashed red), $K_{D M}$ (dashed green), $K_{0}$ (solid red), and, $K_{D}$ (solid green)

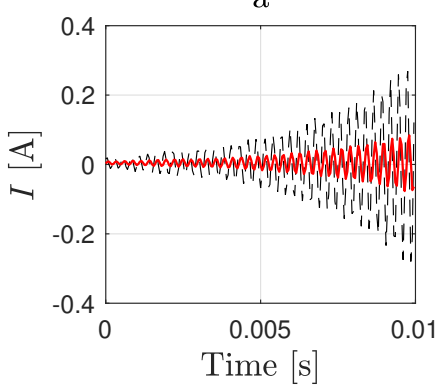

$\mathrm{b}$

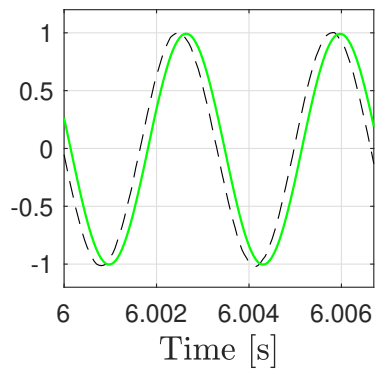

Fig. 18: $I_{R}$ (dashed) and $I_{K}$ (solid) corresponding to scenario No. 8 a) closed-loop instability using $K_{0}$, b) tracking using $K_{D M}$ at $300 \mathrm{~Hz}$

on the feedback current signal, the two-norm of the difference between controller and unity is considered as the objective function. In addition, the constraints corresponding to the stability, robustness, disturbance rejection and the frequency dependent constraints corresponding to maximum allowable signal amplification and attenuation are added to the synthesis problem. These constraints are convexified by linearizing around an initial stabilizing controller, which led to quadratic convex constraints. Since the whole process of data collection and controller design takes roughly less than 30 seconds, the proposed method can be efficiently used as an automatic controller design in PHIL setups. Because the proposed method is in a data-driven framework, there is no need for knowledge about the parameters, order or even structure of the hardware. In addition, the proposed method is extended to a multiscenario case where using one controller, different topologies in HUT or simulation can be tested. The results show that the designed controller can improve the performance of the simulation significantly compared to a conventional low-pass filter. The implementation of the designed controller on the PHIL setup validates the performance of the proposed method.

\section{REFERENCES}

[1] P. A. Lindahl, S. R. Shaw, and S. B. Leeb, "Fuel cell stack emulation for cell and hardware-in-the-loop testing," IEEE Trans. Instrum. Meas., vol. 67, no. 9, pp. 2143-2152, Sept. 2018.

[2] A. Helmedag, T. Isermanna, and A. Monti, "Fault ride through certification of wind turbines based on a hardware in the loop setup," IEEE Trans. Instrum. Meas., vol. 63, no. 10, pp. 2312-2321, Oct. 2014.

[3] M. Matar, H. Karimi, A. Etemadi, and R. Iravani, "A high performance real-time simulator for controllers hardware-in-the-loop testing," Energies, vol. 5, no. 6, pp. 1713-1733, June 2012.

[4] E. Guillo-Sansano, M. H. Syed, A. J. Roscoe, G. M. Burt, and F. Coffele, "Characterization of time delay in power hardware in the loop setups," IEEE Trans. Ind. Electron., vol. 68, no. 3, pp. 2703-2713, Mar. 2021.

[5] J. Langston, T. Szymanski, K. Schoder, M. M. Steurer, and R. G. Roberts, "Practical estimation of accuracy in power hardware-in-theloop simulation using impedance measurements," IEEE Trans. Power Syst., vol. 36, no. 3, pp. 2584-2593, May 2021.

[6] K. Upamanyu and G. Narayanan, "Improved accuracy, modeling, and stability analysis of power-hardware-in-loop simulation with open-loop inverter as power amplifier," IEEE Trans. Ind. Electron., vol. 67, no. 1, pp. 369-378, Jan. 2020.

[7] W. Ren, M. Steurer, and T. L. Baldwin, "An effective method for evaluating the accuracy of power hardware-in-the-loop simulations," IEEE Trans. Appl. Ind., vol. 45, no. 4, pp. 1484-1490, July-aug. 2009. 
[8] F. Lehfuss, G. Lauss, P. Kotsampopoulos, N. Hatziargyriou, P. Crolla, and A. Roscoe, "Comparison of multiple power amplification types for power hardware-in-the-loop applications," in Proc. 2012 Complexity in Engineering (COMPENG), Aachen, Germany, June 2012, pp. 1-6.

[9] N. D. Marks, W. Y. Kong, and D. S. Birt, "Stability of a switched mode power amplifier interface for power hardware-in-the-loop," IEEE Trans. Ind. Electron., vol. 65, no. 11, pp. 8445-8454, Nov. 2018.

[10] W. Ren, M. Steurer, and T. L. Baldwin, "Improve the stability and the accuracy of power hardware-in-the-loop simulation by selecting appropriate interface algorithms," IEEE Trans. Appl. Ind., vol. 44, no. 4, pp. 1286-1294, July-aug. 2008

[11] R. Kuffel et al., "Expanding an analogue hvdc simulator's modelling capability using a real-time digital simulator (RTDS)," in Proc. Int. Conf. Digit. Power Syst. Simulators, Texas, USA, Apr. 1995, pp. 199-204.

[12] X. Wu, S. Lentijo, A. Deshmuk, A. Monti, and F. Ponci, "Design and implementation of a power-hardware-in-the-loop interface: A non-linear load case study," in Proc. IEEE Appl. Power Electron. Conf. and Expo., Austin, USA, Mar. 2005, pp. 1332-1338.

[13] G. F. Lauss, M. O. Faruque, K. Schoder, C. Dufour, A. Viehweider, and J. Langston, "Characteristics and design of power hardware-in-the-loop simulations for electrical power systems," IEEE Trans. Ind. Electron., vol. 63, no. 1, pp. 406-417, Jan. 2016.

[14] A. Viehweider, G. Lauss, and L. Felix, "Stabilization of power hardwarein-the-loop simulations of electric energy systems," Simul. Model Pract. Theory, vol. 19, no. 7, pp. 1699-1708, Aug. 2011.

[15] G. Lauss and K. Strunz, "Accurate and stable hardware-in-the-loop (HIL) real-time simulation of integrated power electronics and power systems," IEEE Trans. Power Electron., vol. 36, no. 9, pp. 1092010932, Sept. 2021.

[16] A. Summers, J. Hernandez-Alvidrez, R. Darbali-Zamora, M. J. Reno, J. Johnson, and N. S. Gurule, "Comparison of ideal transformer method and damping impedance method for PV power-hardware-in-the-loop experiments," in Proc. IEEE Photovolt. Specialists Conf., Chicago, USA, June 2019, pp. 2989-2996.

[17] W. Ren et al., "Interfacing issues in real-time digital simulators," IEEE Trans. Power Del., vol. 26, no. 2, pp. 1221-1230, Apr. 2011.

[18] S. Ayasun, R. Fischl, S. Vallieu, J. Braun, and D. Çadirli, "Modeling and stability analysis of a simulation-stimulation interface for hardware-inthe-loop applications," Simul. Model Pract. Theory, vol. 15, no. 6, pp. 734-746, July 2007.

[19] M. G. Kashani, H. Pulakhandam, S. Bhattacharya, F. Katiraei, and D. Kaiser, "Design considerations and test setup assessment for power hardware in the loop testing," in Proc. IEEE Ind. Appl. Soc. Annu. Meeting, Cincinnati, USA, Oct. 2017, pp. 1-8.

[20] T. Reinikka, H. Alenius, T. Roinila, and T. Messo, "Power hardware-inthe-loop setup for stability studies of grid-connected power converters," in Proc. Int. Power Electron. Conf., IPEC, Niigata, Japan, May 2018, pp. 1704-1710.

[21] M. Bokal, I. Papic, and B. Blazic, "Stabilization of hardware-in-theloop ideal transformer model interfacing algorithm by using spectrum assignment," IEEE Trans. Power Del., vol. 34, no. 5, pp. 1865-1873, Oct. 2019.

[22] L. Ljung, System Identification - Theory for the User (2nd edn). Prentice Hall: NJ, USA, 1999.

[23] Z. S. Hou and Z. Wang, "From model-based control to data-driven control: Survey, classification and perspective," Information Sciences, vol. 235, pp. 3-35, June 2013.

[24] X. Wang, J. Sun, J. Berberich, G. Wang, F. Allgöwer, and J. Chen, "Data-driven control of dynamic event-triggered systems with delays," Oct. 2021. [Online]. Available: http://arxiv.org/abs/2110.12768

[25] C. D. Persis and P. Tesi, "Formulas for data-driven control: Stabilization, optimality, and robustness," IEEE Trans. Autom. Control, vol. 65, no. 3, pp. 909-924, Mar. 2020.

[26] M. Guo, C. D. Persis, and P. Tesi, "Data-driven stabilization of nonlinear polynomial systems with noisy data," IEEE Trans. Autom. Control, 2021, early access. doi: 10.1109/TAC.2021.3115436.

[27] P. Vuillemin, P. Kergus, and C. Poussot-Vassal, "Hybrid loewner data driven control," in Proc. 21st IFAC World Congress, July 2020, pp. 5604-5608.

[28] P. Kergus, "Data-driven control of infinite dimensional systems: Application to a continuous crystallizer," IEEE Control Systems Letters, vol. 5, no. 6 , pp. $2120-2125$, Dec. 2021

[29] N. Nikooienejad and S. O. R. Moheimani, "Convex synthesis of SNI controllers based on frequency-domain data: MEMS nanopositioner example," IEEE Trans. Control Syst. Technol., 2021, early access. 10.1109/TCST.2021.3080577.
[30] A. Karimi and C. Kammer, "A data-driven approach to robust control of multivariable systems by convex optimization," Automatica, vol. 85, pp. 227-233, Nov. 2017

[31] T. Bloemers, R. Tóth, and T. Oomen, "Frequency-domain data-driven controller synthesis for unstable LPV systems," in Proc. 4th IFAC Workshop on Linear Parameter Varying Systems, Milan, Italy, July 2021, pp. 109-115.

[32] O. Bagherieh, P. Shah, and R. Horowitz, "Mixed $\mathrm{H}_{2} / \mathrm{H}_{\infty}$ data-driven control design for hard disk drives," in Proc. Asia-Pacific Magnetic Recording Conf., Shanghai, China, Nov. 2018, pp. 1-3.

[33] J. Pan, Z. Chen, Y. Wang, and R. Horowitz, "Frequency separation based adaptive feedforward control for rejecting wideband vibration with application to hard disk drives," Dec. 2020. [Online]. Available: http://arxiv.org/abs/2012.05049

[34] M. Pokharel and C. N. M. Ho, "Stability analysis of power hardwarein-the-loop architecture with solar inverter," IEEE Trans. Ind. Electron., vol. 68, no. 5, pp. 4309-4319, May 2021.

[35] O. Nzimako and R. Wierckx, "Stability and accuracy evaluation of a power hardware in the loop (PHIL) interface with a photovoltaic microinverter," in Proc. IEEE IECON 2015, Yokohama, Japan, Nov. 2015, pp. 5285-5291.

[36] M. Hong, S. Horie, Y. Miura, T. Ise, and C. Dufour, "A method to stabilize a power hardware-in-the-loop simulation of inductor coupled systems," in Proc. IEEE Int. Conf. Power Syst. Transients, Kyoto, Japan, Jun. 2009, pp. 1-7.

[37] A. Riccobono, E. Liegmann, M. Pau, F. Ponci, and A. Monti, "Online parametric identification of power impedances to improve stability and accuracy of power hardware-in-the-loop simulations," IEEE Trans. Instrum. Meas., vol. 66, no. 9, pp. 2247-2257, Sept. 2017.

[38] E. Liegmann, A. Riccobono, and A. Monti, "Wideband identification of impedance to improve accuracy and stability of power-hardware-inthe-loop simulations," in Proc. Int. Workshop Appl. Meas. Power Syst. Aachen, Germany, Sep. 2016, pp. 1-6.

[39] J. Siegers and E. Santi, "Improved power hardware-in-the-loop interface algorithm using wideband system identification," in Proc. Appl. Power Electron. Conf. Expo., Fort Worth, USA, Mar. 2014, pp. 1198-1204.

[40] —, "Stability analysis and control design for an all-electric ship MVDC power distribution system using a passivity based stability criterion and power hardware-in-the-loop simulation," in Proc. Electric Ship Technol. Symp., Alexandria, USA, June 2015, pp. 86-92.

[41] T. Roinila, H. Abdollahi, S. Arrua, and E. Santi, "Adaptive control of DC power distribution systems: Applying pseudo-random sequences and fourier techniques," in Proc. Int. Power Electron. Conf. Asia, Niigata, Japan, May 2018, pp. 1719-1723.

[42] S. Boyd and L. Vandenberghe, Convex Optimization. Cambridge University Press, 2004.

[43] M. ApS, The MOSEK optimization toolbox for MATLAB manual. Version 9.3., 2021. [Online]. Available: http://docs.mosek.com/9.3/ toolbox/index.html

[44] M. Dargahi, A. Ghosh, and G. Ledwich, "Stability synthesis of power hardware-in-the-loop (PHIL) simulation," in Proc. Power Energy Soc. General Meeting, National Harbor, USA, July 2014, pp. 1-5.

[45] G. Lauss, F. Lehfuß, A. Viehweider, and T. Strasser, "Power hardware in the loop simulation with feedback current filtering for electric systems," in Proc. Ind. Electron. Conf., Melbourne, Australia, Nov. 2011, pp. $3725-3730$

[46] G. Lauss and K. Strunz, "Multirate partitioning interface for enhanced stability of power hardware-in-the-loop real-time simulation," IEEE Trans. Ind. Electron., vol. 66, no. 1, pp. 595-605, Jan. 2019.

[47] A. Riccobono, A. Helmedag, A. Berthold, N. R. Averous, R. W. De Doncker, and A. Monti, "Stability and accuracy considerations of power hardware- in-the-loop test benches for wind turbines," in Proc. 20th IFAC World Congress, vol. 50, Toulouse, France, Jul. 2017, pp. 10 977-10 984.

[48] N. Ainsworth, A. Hariri, K. Prabakar, A. Pratt, and M. Baggu, "Modeling and compensation design for a power hardware-in-the-loop simulation of an AC distribution system," in Proc. 48th North American Power Symp., Denver, USA, Sept. 2016, pp. 1-6.

[49] S. S. Madani and A. Karimi, "Data-driven LPV controller design for islanded microgrids," in Proc. 19th IFAC Symposium on System Identification SYSID 2021, July 2021, pp. 433-438.

[50] S. Winder, Analog and Digital Filter Design, 2nd ed. Newnes, 2002.

[51] R. Pintelon and J. Schoukens, System identification: a frequency domain approach. IEEE Press, 2001.

[52] A. Markou, V. Kleftakis, P. Kotsampopoulos, and N. Hatziargyriou, "Improving existing methods for stable and more accurate power hardwarein-the-loop experiments," in Proc. Int. Symp. Ind. Electron., Edinburgh, UK, June 2017, pp. 496-502. 
[53] A. Karimi, A. Nicoletti, and Y. Zhu, "Robust $\mathrm{H}_{\infty}$ controller design using frequency-domain data via convex optimization," International Journal of Robust and Nonlinear Control, vol. 28, pp. 3766-3783, July 2018.

[54] X. Song et al., "Research on performance of real-time simulation based on inverter-dominated power grid," IEEE Access, vol. 9, pp. 1137-1153, 2021.

[55] IEEE PES Distribution Systems Analysis Subcommittee Radial Test Feeders, IEEE Std. [Online]. Available: https://cmte.ieee.org/ pes-testfeeders/resources/

Seyed Sohail Madani received the M.Sc. degree in Electrical Engineering from Sharif University of Technology, Tehran, Iran in 2012. He is currently pursuing a Ph.D. at École Polytechnique Fédérale de Lausanne (EPFL), Lausanne, Switzerland. His research interests include power electronics and microgrid simulation and control.

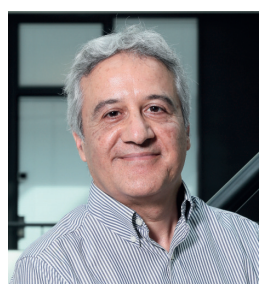

Alireza Karimi received the Ph.D. degree in 1997 from Institut National Polytechnique de Grenoble, France. He was an Assistant Professor with the Department of Electrical Engineering, Sharif University of Technology, Tehran, Iran, from 1998 to 2000 He Joined then Ecole Polytechnique Fédérale de Lausanne (EPFL) in Switzerland and is currently a Professor of automatic control in the Mechanical Engineering Institute of EPFL. Prof. Karimi is the head of "data-driven modelling and control" group in Automatic Control Laboratory and his research interests include data-driven controller design approaches with applications to power grids and mechatronic systems. Prof. Karimi was an Associate Editor of European Journal of Control from 2004 to 2013 and is a member of the conference editorial board of IEEE control systems society since 2018. 